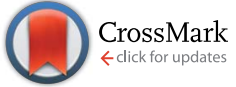

Cite this: RSC Adv., 2017, 7, 13760

Received 12th November 2016 Accepted 11th February 2017

DOI: 10.1039/c6ra26713k

rsc.li/rsc-advances

\section{A novel nano-sized bioactive glass stimulates osteogenesis via the MAPK pathway}

\author{
Weiyu Gong, ${ }^{a}$ Yanmei Dong, ${ }^{\text {*a }}$ Sainan Wang, ${ }^{a}$ Xuejun Gao and Xiaofeng Chen ${ }^{\mathrm{b}}$
}

The aims of this study were to compare the osteogenic effects of a novel nano-sized bioactive glass (BG) and a traditional micron-sized BG, and to verify whether mitogen-activated protein kinases (MAPKs) are involved and play a part in BG's osteoblast gene activation. It was found that the effect of a nano-sized $\mathrm{BG}$ on MAPK phosphorylation is better than the traditional $45 \mathrm{~S} 5 \mathrm{BG}$. We prepared extractions of the novel nano-sized 58S BG and traditional 45S5 BG and compared their effect on osteoblast-like cells' (MG-63 cell) proliferation, osteogenic gene and protein expressions, matrix mineralization and MAPK activation. We further investigated the signal transducing effect of the MAPK pathway on the BG's osteogenic gene activation. Our results showed that nano-58S extraction enhanced the MG-63 cells' proliferation and osteogenic gene expressions of alkaline phosphatase (ALP), collagen type I (Col I), Runt-related transcription factor 2 (Runx2) and osteocalcin (OCN). The results of ELISA showed more Col I and OCN protein production in the BG groups than in the control group. Greater mineralized nodule formation was found in the nano-58S BG group using alizarin red $\mathrm{S}$ staining. We also found that nano-58S and 45S5 BG activated MAPKs, specifically the ERK and p38 pathways, using western blotting. After blocking the ERK or p38 pathway, real-time PCR showed the osteogenic gene activation induced by the BG extractions was inhibited. Blocking the ERK pathway induced a more obvious inhibitory effect on the genes' normal expression and activation. The significance of this study is that we found that the ERK and p38 MAPK pathways are involved and play an important part in BG's osteogenic gene activation. The effect of the nano-sized BG on MAPK phosphorylation, osteogenic gene activation, and osteoblast differentiation and mineralization is better than the traditional 4555 BG.

\section{Introduction}

The demands on osteoinductive biomaterials have been rapidly growing in recent years due to bone defects, which are common, caused by trauma, post-cancer surgery, periodontal disease and other skeletal diseases. Bioactive glass (BG) is a kind of highly biocompatible, osteoinductive and osteoconductive calcium silicate-based biomaterial. ${ }^{1}$ Since it was invented in the 1970s, BG has been used in the clinical treatment of periodontitis, ${ }^{2}$ maxillary cystic bone defects and implantation ${ }^{3}$ for almost 30 years and proven to have strong direct bonds to bone tissue and excellent osteogenic effects. ${ }^{\mathbf{1 , 4 - 6}}$ Recently, several investigators have found that nano-sized sol-gel BG possesses higher specific surface area, better bioactivity and induces more osteoblast adhesion compared to traditional micron-sized BG particles. ${ }^{7-9}$

Studies have proven that BG regulates the expression of several genes related to cell proliferation, ${ }^{6}$ differentiation, and bone matrix formation such as alkaline phosphatase (ALP),

${ }^{a}$ Department of Cariology and Endodontology, Peking University School and Hospital of Stomatology, 22 Zhongguancun Nandajie, Haidian District, Beijing 100081, China. E-mail: kqdongyanmei@bjmu.edu.cn; Fax: +86-10-62173402; Tel: +86-10-82195385

${ }^{b}$ National Engineering Research Center for Human Tissue Restoration \& Reconstruction, South China University of Technology, Guangzhou, China bone sialoprotein (BSP), Runt-related transcription factor 2 (Runx2) and osteocalcin (OCN)., ${ }^{\mathbf{4 , 5 1 0 - 1 2}}$ However, there are still many questions to be answered about the molecular mechanism of BG's regulation of the osteoblasts' gene expression.

The mitogen-activated protein kinase (MAPK) family, which is composed of branches of extracellular signal-regulated protein kinases (ERK), p38, c-Jun N-terminal kinase (JNK) and ERK5, plays an important role in cell proliferation, differentiation, apoptosis and stress response. ${ }^{\mathbf{1 3 - 1 5}}$ Several studies have found that the gene expression of MAPKs changed when cells were cultured with BG. ${ }^{12,16,17}$ On the other hand, silicon (Si) and calcium $(\mathrm{Ca})$ in high concentrations can activate MAPKs. ${ }^{\mathbf{1 8 - 2 0}}$ Meanwhile, Si and $\mathrm{Ca}$ ions are also indispensable in BG's bioactivity. ${ }^{16}$ So far, the previous studies have found that BG affects the gene expression of MAPKs, however, little is known about whether BG regulates gene expression via the MAPK pathway and the gene regulation mechanism.

MG-63 cells are osteoblast-like cell lines isolated from a human osteosarcoma and they possess the typical characteristics of a series of osteoblast phenotypes, including the expression of BMP 2, ALP, Runx2 and OCN. Although MG-63 cells can be infinitely passaged, this cell line as an ideal 
replacement of primary osteoblast cells is widely used in studies on osteogenesis. ${ }^{21,22}$

Besides, due to their involvement in the regulation of cells' proliferation, differentiation, and apoptosis, the function and performance of MAPKs are affected by cell viability. The expression of MAPKs often changes because of different passages' cell viability and individual differences. Therefore, it is difficult to investigate the real and stable performance of MAPKs in the different passages of primary osteoblasts. On the other hand, because of their infinite passage capacity, MG-63 cells show similar and stable viability and function performance like osteoblasts. The stable and repeatable osteogenesisrelated MAPK activation can be observed in this cell line.

The aim of the present study is to verify two hypotheses: (1) MAPKs are involved and play a role in BG's regulation of osteoblasts' gene expression; and (2) the effects of nano-sized 58S BG on osteogenic gene up-regulation and MAPK phosphorylation are higher than the traditional 45S5 BG.

\section{Materials and methods}

\subsection{Characteristics of BG}

Nano-58S BG (58\% $\mathrm{SiO}_{2}, 33 \% \mathrm{CaO}$ and $\left.9 \% \mathrm{P}_{2} \mathrm{O}_{5}(\mathrm{w} / \mathrm{w})\right)$ and traditional melt $45 \mathrm{~S} 5 \mathrm{BG}\left(45 \% \mathrm{SiO}_{2}, 24.5 \% \mathrm{CaO}, 6 \% \mathrm{P}_{2} \mathrm{O}_{5}\right.$ and $\left.24.5 \% \mathrm{Na}_{2} \mathrm{O}(\mathrm{w} / \mathrm{w})\right)$ were prepared by the National Engineering Research Center for Human Tissue Restoration \& Reconstruction, South China University of Technology (Guangzhou, China). The preparation methods for the novel nano-58S BG were described previously. ${ }^{23}$ Both of the bioactive glasses were mainly composed of amorphous silicate phases. ${ }^{24,25}$ The surface morphology of the particulates was observed by field emission scanning electron microscopy (FE-SEM) (S4800, JEOL, Tokyo, Japan).

\subsection{BG extraction}

The BG particulates were sterilized in a dry heat oven at $180{ }^{\circ} \mathrm{C}$ for $4 \mathrm{~h}$. BG extractions were prepared by incubating nano-58S or 45S5 BG in Dulbecco's Modified Eagle Medium (DMEM) (Gibco, Gaithersburg, MD, USA) at a concentration of $1 \mathrm{mg} \mathrm{mL} \mathrm{m}^{-1}$ at $37{ }^{\circ} \mathrm{C}$ for $24 \mathrm{~h}$. The mixtures were centrifuged and the supernatant was filtrated through a $0.22 \mu \mathrm{m}$ filter (Millipore, Billerica, MA, US). All BG extractions were supplemented with $10 \%$ fetal bovine serum (Hyclone, Logan, UT, USA), $100 \mathrm{mg} \mathrm{mL} \mathrm{mL}^{-1}$ streptomycin, $100 \mathrm{U} \mathrm{mL}^{-1}$ penicillin and $2 \mathrm{mM}$ L-glutamine. The $\mathrm{pH}$ of the BG extractions was adjusted to 7.4 for cellular experiments. The concentrations of $\mathrm{Si}, \mathrm{Ca}$, and phosphorus (P) in the extractions were detected using inductively coupled plasma optical emission spectroscopy (ICP-OES) analysis (iCAP 6300, Thermo Electron Corporation, Waltham, MA, USA).

\subsection{Cell proliferation assay}

The MG-63 osteoblast-like cells used in this study were obtained from the American Type Culture Collection (ATCC No. CRL 1427). The cells were cultured with the extractions of nano-58S BG, 45S5 BG and growth medium (control group) for 10 days.
Cell proliferation was determined using the methyl-thiazoltetrazolium (MTT) (Amresco, Solon, OH, USA) method.

\subsection{Real-time polymerase chain reaction (PCR)}

The total RNA of the MG-63 cells was extracted using Trizol® reagent (Invitrogen, Carlsbad, CA, US) and reversely transcribed into complementary DNA (cDNA) by reverse transcriptase (Promega, Madison, WI, USA) according to the manufacturers' instructions.

Real-time PCR was performed using a Faststart Universal SYBR Green PCR Master mix (Roche, Indianapolis, IN, US) and Applied Biosystem 7500 thermal cycler (ABI 7500, Applied Biosystems, Foster City, CA, US). Real-time PCR was performed with primers designed by Primer 5.0 according to the cDNA sequences available in the GenBank database for ALP (F: 5' AGCACTCCCACTTCATCTGGAA-3', R: 5'-GAGACCCAATAGGT AGTCCACATTG-3'), collagen I (Col I, F: 5'-CGAAGACATCCC ACCAATCAC-3', R: 5'-TGTCGCAGACGCAGAT-3'), Runx2 (F: 5'ACCCAGAAGGCACAGACAGAAG-3', R: 5'-AGGAATGCGCCCTAA ATCACT-3') and OCN (F: 5'-AGGGCAGCGAGGTAGTGA-3', R: 5' CCTGAAAGCCGATGTGGT-3'). Glyceraldehyde 3-phosphate dehydrogenase (GAPDH, F: 5'-CAACGGATTTGGTCGTATTGG-3', R: 5'-GCAACAATATCCACTTTACCAGAGTTAA-3') was used for the quantification of relative gene expression. The thermal cycling conditions were as follows: $50{ }^{\circ} \mathrm{C}$ for $2 \mathrm{~min}$ and $95^{\circ} \mathrm{C}$ for $10 \mathrm{~min}$, and 40 cycles of $94{ }^{\circ} \mathrm{C}$ for $15 \mathrm{~s}$ and $60^{\circ} \mathrm{C}$ for $1 \mathrm{~min}$.

\subsection{Immunocytochemical assay of Runx 2 and OCN}

Cells were washed with phosphate buffer solution (PBS) and fixed with $4 \%$ paraformaldehyde (Sigma, St. Louis, MO, USA). After being punched with $0.1 \%$ Triton $\mathrm{X}-100$ and immersed in $3 \% \mathrm{H}_{2} \mathrm{O}_{2}$, the cells were blocked with $0.5 \%$ goat serum. Afterwards, rabbit anti-human Runx 2 and OCN primary antibody (dilution 1 : 300; Santa Cruz, CA, US) were added overnight at $4{ }^{\circ} \mathrm{C}$. Subsequently, the cells were incubated with goat antirabbit secondary antibody and were subject to enzymatic immunohistochemical staining using a broad-spectrum immunoperoxidase diaminobenzidine kit (Zhongshan Bioengineering, Beijing, China). Finally, the cells were counterstained with hematoxylin and visualized.

\subsection{Enzyme-linked immunosorbent assay (ELISA) of Col I and $\mathrm{OCN}$}

The MG-63 cells were cultured with the extractions of nano-58S BG, 45S5 BG and growth medium (control group) for 4 days and 7 days. The media were collected and stored at $-80^{\circ} \mathrm{C}$ until use. Protein expression levels of Col I and OCN in the culture supernatant were assayed using a human Col I and OCN enzyme-linked immunosorbent assay kit (R\&D Systems, Minneapolis, MN) according to the manufacturer's instructions. The protein concentration of OCN was calculated from a calibration curve using human $\mathrm{Col}$ I and OCN concentrations as the standard. 


\subsection{Matrix mineralization assay}

The MG-63 cells were cultured in the BG extractions with an osteogenic medium (10 mmol L ${ }^{-1} \beta$-glycerophosphate, $10 \mathrm{nmol}$ $\mathrm{L}^{-1}$ dexamethasone, and $50 \mathrm{mg} \mathrm{L}^{-1}$ ascorbic acid) for 28 days. The cells cultured in the growth medium were used as the nonosteogenic control group; those cultured in the growth medium with the osteogenic medium were assessed as the osteogenic control group. For the matrix mineralization assay, the cells were rinsed 3 times with PBS and fixed in $4 \%$ paraformaldehyde for 20 minutes. The cells were washed using deionized water 3 times and then stained with $40 \mathrm{mmol} \mathrm{L}^{-1}$ alizarin red S solution (pH 4.2) (Sigma-Aldrich, St. Louis, MO, US) and observed for mineralized nodules.

For matrix mineralization semi-quantification, $1 \mathrm{~mL}$ of $100 \mathrm{mM}$ cetylpyridinium chloride (Sigma-Aldrich, St. Louis, MO, US) was used to dissolve the dried alizarin red S stained cellular matrix. The optical density was detected using the absorbance at $562 \mathrm{~nm}$.

\subsection{Extraction of proteins and western blotting}

Cells were washed with ice-cold PBS and then lysed using RIPA cell lysis buffer (Cell Signaling Technology, Beverly, MA, US). Then the mixtures were centrifuged at $14000 \mathrm{~g}$ for $20 \mathrm{~min}$ at $4{ }^{\circ} \mathrm{C}$. The concentration of protein in the supernatant was determined using a BCA Protein Assay Kit (Pierce, Rockford, IL, USA). For western blot analysis, protein samples $(20 \mu \mathrm{g})$ underwent $10 \%$ SDS-PAGE (electrophoretic migration at $100 \mathrm{~V}$ ) and were electrotransferred onto polyvinylidene fluoride (PVDF) membranes. After being incubated in a blocking solution (50 mM Tris/ $\mathrm{HCl}, 200 \mathrm{mM} \mathrm{NaCl}, 0.2 \%$ Tween 20 , and $5 \%$ nonfat dried milk), the PVDF membranes were incubated in the presence of $1: 1000$ diluted primary monoclonal rabbit anti-human phosphorylated (p-)ERK, anti-human phosphorylated (p-)JNK, anti-human phosphorylated (p-)p38 (Cell Signaling Technology) and $\beta$-actin (Santa Cruz, CA, USA) antibodies at $4{ }^{\circ} \mathrm{C}$ overnight. After washing with TRIS-buffered saline (0.2\% Tween 20), the membranes were incubated with 1 : 10000 diluted mouse antirabbit secondary antibody (Cell Signaling Technology). The immunoreactive bands were visualized using an ECL detection reagent (Thermo Fisher, Waltham, MA, US), exposed to Kodak $\mathrm{X}$-ray films and quantitatively analyzed using Image $1.38(\mathrm{NIH})$. After washing in stripping buffer, the same transferred membrane was tested for the total protein expression with rabbit anti-human non-phosphorylated MAPK antibodies (Cell Signaling Technology).

To confirm whether osteogenic gene activation by BG extraction is mediated through MAPK pathways, we added 20 $\mu \mathrm{M}$ ERK1/2 inhibitor (U0126) (Cell Signaling Technology) or p38 inhibitor (SB203580) (Calbiochem, Billerica, MA, US) during culturing of the cells, and observed the change in BG-induced gene activation at day 4 .

\section{Statistical analysis}

Each experiment was run in triplicate, and each result was averaged using at least three parallel samples and measurements. All data are expressed as mean \pm standard deviation (SD). Variance and statistical significance were determined using Student's $t$-test using SPSS Statistics 17.0 software. Differences were considered significant at $P<0.05$.

\section{Results}

\subsection{Characteristics of $B G$}

FE-SEM showed that the nano-58S BG particulates were regular and spherical with sizes of approximately $20 \mathrm{~nm}$ (Fig. 1(a)), while the shape of the 45S5 BG particulates was irregular with sizes ranging between 2 and $15 \mu \mathrm{m}$ (Fig. 1(b)).

According to the results of the ICP-OES analysis, the highest concentrations of $\mathrm{Ca}$ and $\mathrm{Si}$ were detected in the nano-58S BG extraction compared to the control and 45S5 BG groups. The concentration of $\mathrm{P}$ was lower in the nano-58S BG and 45S5 BG extractions than in the control group (Fig. 1(c)).

\subsection{Effect of BG extractions on MG-63 cells proliferation and osteogenic genes expression}

The MTT results showed that BG enhanced cell proliferation (Fig. 1(d)). The $\mathrm{OD}_{490}$ values of the two BG groups were both significantly higher than that of the control group $(P<0.05)$.

The mRNA expression of ALP, Col I, Runx2 and OCN are shown in Fig. 1(e)-(h). Compared with the control group, ALP gene expression was significantly up-regulated by nano-58S and 45S5 BG extractions at 2 and 4 days, and the expression in the nano-58S group was significantly higher than that of the $45 \mathrm{~S} 5$ group at 4 days. The mRNA expression of Col I at 4 days and 7 days, and Runx 2 at 2, 4 and 7 days in the nano-58S group was significantly higher than those of the 45S5 group and the control group. 45S5 BG up-regulated Runx2 gene expression significantly at 2 days and 4 days, but with no difference at 7 days. Nano-58S BG induced significantly higher OCN gene expression at 4 days and 7 days than 45S5 BG and the control.

The immunocytochemical assay of Runx2 at day 4 (Fig. 2(a)) and OCN at day 7 (Fig. 2(b)) showed the most intense positive staining of both proteins in the nano-58S group. Moderate and negative staining were found in the 45S5 group and control group, respectively.

ELISA quantified the protein secretion of Col I and OCN, and determined whether the changes in Col I and OCN protein levels were in parallel with gene activation. In agreement with the observed increase in RNA levels, nano-58S BG enhanced Col I and OCN protein secretion compared to 45S5 BG and the control group on day $7(P<0.05)$ (Fig. 2(c) and (d)).

The mineralization assay indicated that nano-58S significantly promoted mineral nodule formation (Fig. 3(a)). At 28 days, no red staining was observed in the non-osteogenic control group and moderate red nodule staining was observed in the osteogenic control group. As for the BG groups, the density of mineralized nodules was significantly higher in the nano-58S group than in the other groups. The 45S5 group showed similar staining to the osteogenic control group. The 
(a)

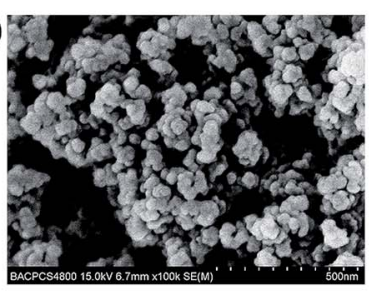

(b)

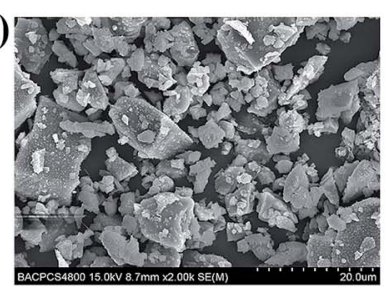

(c)

\begin{tabular}{llll}
\hline & $\mathrm{Si}(\mu \mathrm{g} / \mathrm{mL})$ & $\mathrm{Ca}(\mu \mathrm{g} / \mathrm{mL})$ & $\mathrm{P}(\mu \mathrm{g} / \mathrm{mL})$ \\
\hline DNEM & 0.57 & 75.33 & 29.18 \\
(control) & & 88.88 & 13.34 \\
$45 \mathrm{~S} 5$ & 44.23 & 154.50 & 1.72 \\
nano-58S & 71.00 & 154 \\
\hline
\end{tabular}

(d)

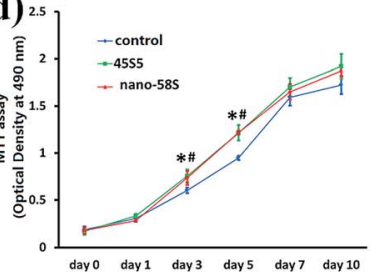

(e)

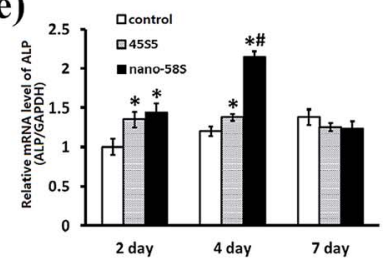

(f)

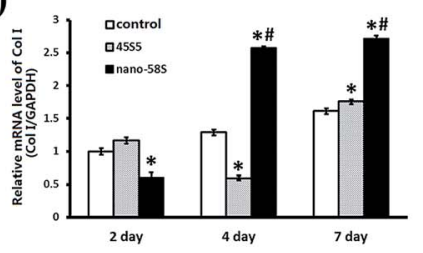

(g)

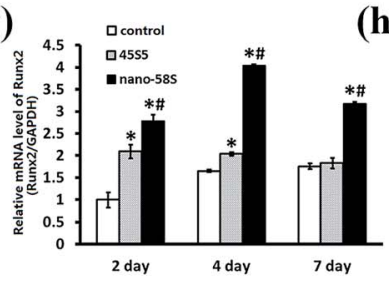

(h)

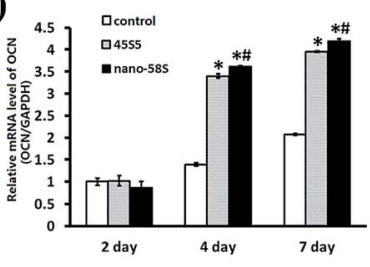

Fig. 1 (a) Surface morphology of the nano-58S particulates observed using FE-SEM. (b) Surface morphology of the 45S5 particulates observed using FE-SEM. (c) Concentrations of Si, Ca and P in the BG extractions. (d) Effects of nano-58S and 45S5 BG extractions on MG-63 cell proliferation. (e)-(h) Real-time PCR results of differentiation and mineralization gene expression. The data are relative expression levels shown as the fold difference compared with the control group at day 1 and are normalized to the expression level of the housekeeping gene (GAPDH). The data are the mean values \pm standard deviation of three individual experiments. Real-time PCR experiments were run twice for each sample (ANOVA, ${ }^{*} P<0.05$ versus the control group; ${ }^{\#} P<0.05$ versus the 4555 group).

results of the semi-quantification of $\mathrm{Ca}$ deposition were consistent with the mineralization staining (Fig. 3(b)). The $\mathrm{OD}_{562}$ of the nano-58S group was significantly higher than the osteogenic control group. No statistical difference between the $\mathrm{OD}_{562}$ values was found between the $45 \mathrm{~S} 5$ group and the osteogenic control groups.

\subsection{Effects of BG extractions on MAPK activation}

The results of western blotting showed that BG extractions significantly enhanced the phosphorylation of ERK and p38 at $12 \mathrm{~h}$, and 1 and 3 days (Fig. 4(a)). Quantified using ImageJ 1.38 software (Fig. 4(b)), the expression of p-ERK was significantly higher in the nano-58S group than in the 45S5 and control

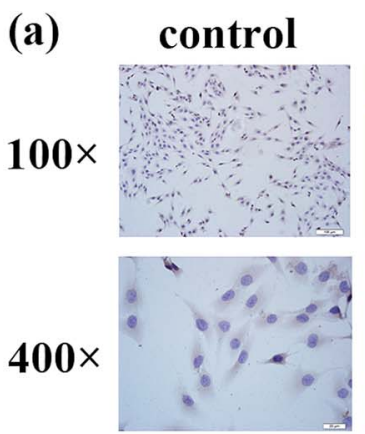

(b)

$100 \times$

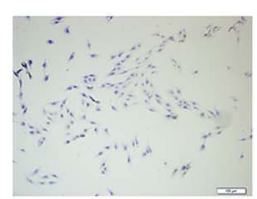

$400 \times$

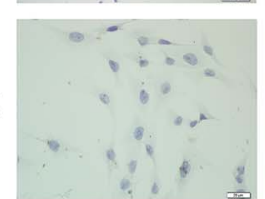

$45 S 5$
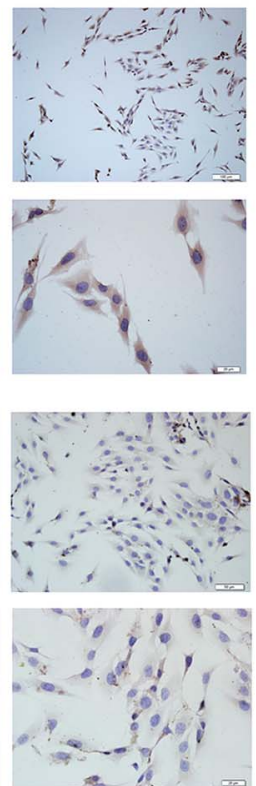

nano-58S
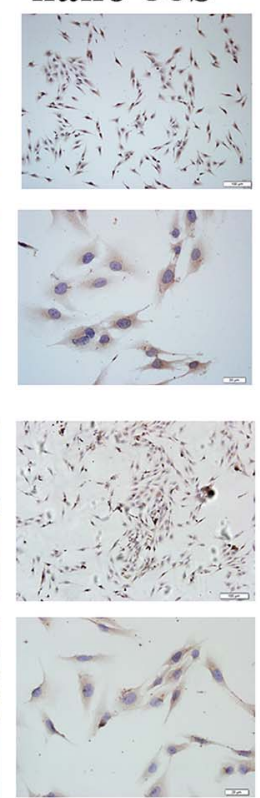

(c)

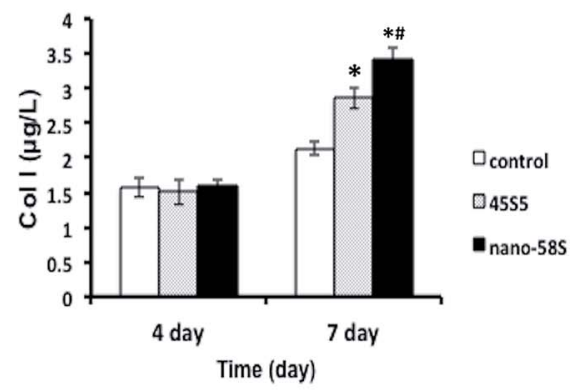

(d)

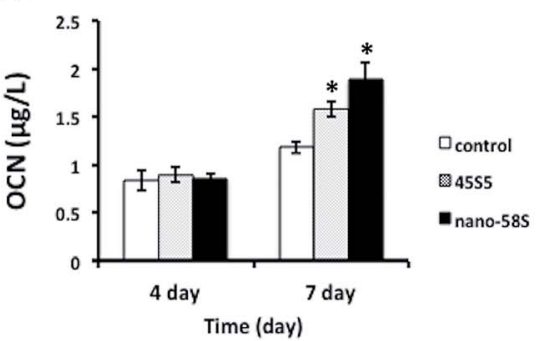

Fig. 2 (a) Immunocytochemical staining of Runx2 at day 4; (b) immunocytochemical staining of OCN at day 7; (c) ELISA of Col I at day 4 and day 7; and (d) ELISA of OCN at day 4 and day 7 (ANOVA, ${ }^{*} P<0.05$ versus the control group; ${ }^{*} P<0.05$ versus the $45 \mathrm{~S} 5$ group). 
(a)
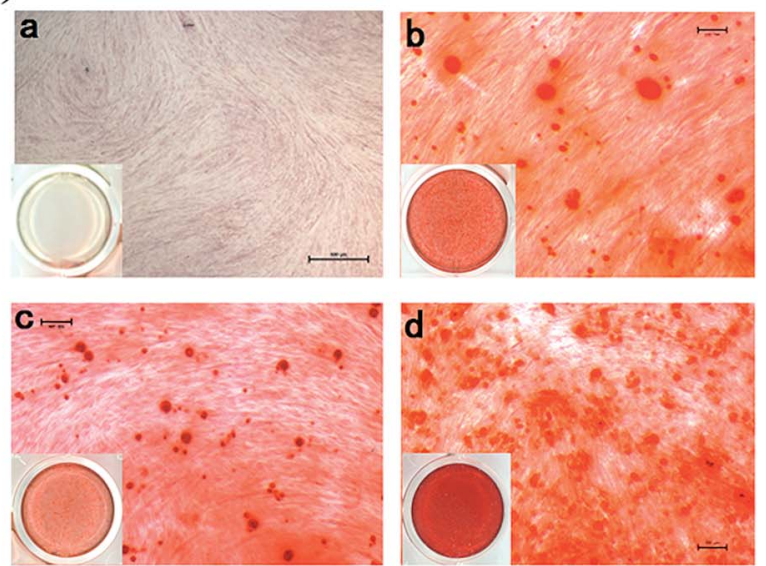

(b)

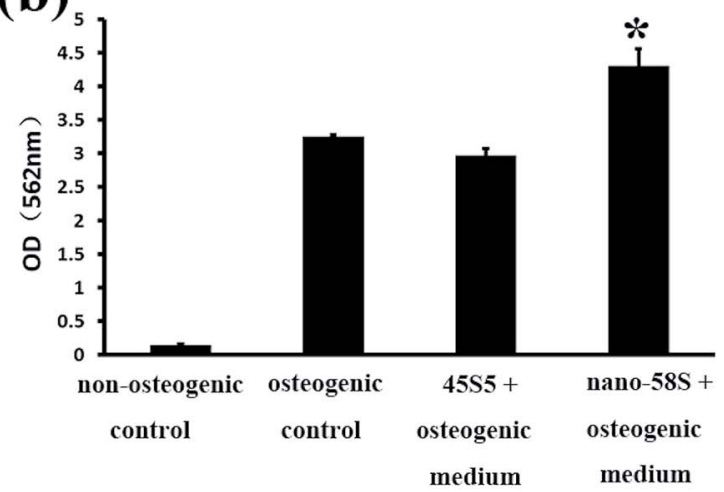

Fig. 3 (a) Alizarin S staining of the mineralized nodules of MG-63 cells cultured in a: non-osteogenic control medium, b: osteogenic control medium, c: 4555 BG extraction with osteogenic medium and d: nano$58 \mathrm{~S}$ BG extraction with osteogenic medium at day 28; and (b) semiquantification of calcium deposits of MG-63 cells cultured in nano$58 \mathrm{~S}$ and 45S5 BG extractions and a control medium at day 28 (ANOVA, $* P<0.05$ versus the osteogenic control group).

groups. The expression of p-p38 in the nano-58S group was significantly up-regulated at 12 hours, and 1 and 3 days compared with the control group. The expression of p-p38 in the $45 \mathrm{~S} 5$ group was similar to the nano-58S group at 12 hours and then showed no significant up-regulation compared to the control group. No expression change of JNK or p-JNK was found throughout the observations.

When the ERK pathway was blocked, the normal expressions of ALP, Runx2, Col I and OCN were inhibited significantly. With the ERK pathway blocked, nano-58S still increased ALP gene expression significantly compared to $45 \mathrm{~S} 5$ and the control group but it was significantly lower than the normal expression levels of the control group without U0126, also Col I gene expression in the 45S5 group was significantly lower than in the control and nano$58 \mathrm{~S}$ groups. With U0126, the mRNA levels of Col I, Runx 2 and OCN in the nano-58S group and ALP, Runx 2 and OCN in the 45S5 group were similar to the ERK blocked control group, which indicated no significant gene activation (Fig. 5(a)). When the p38 pathway was blocked, the gene activation of ALP showed no (a)

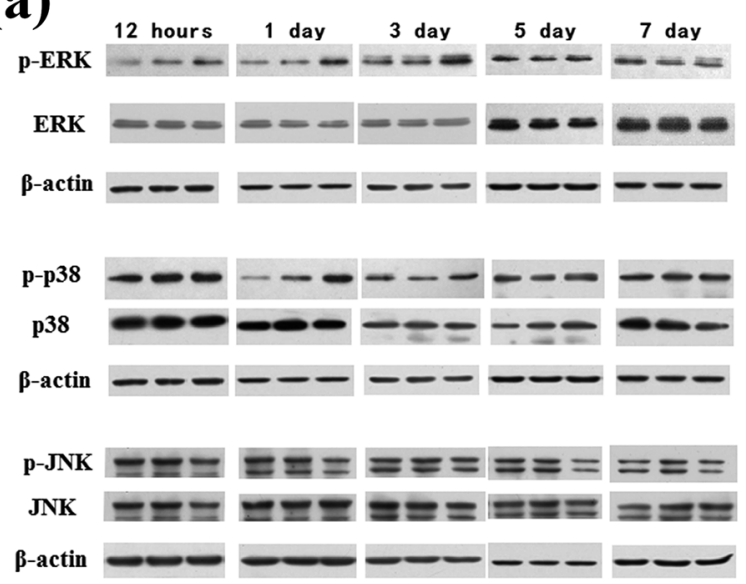

(b)
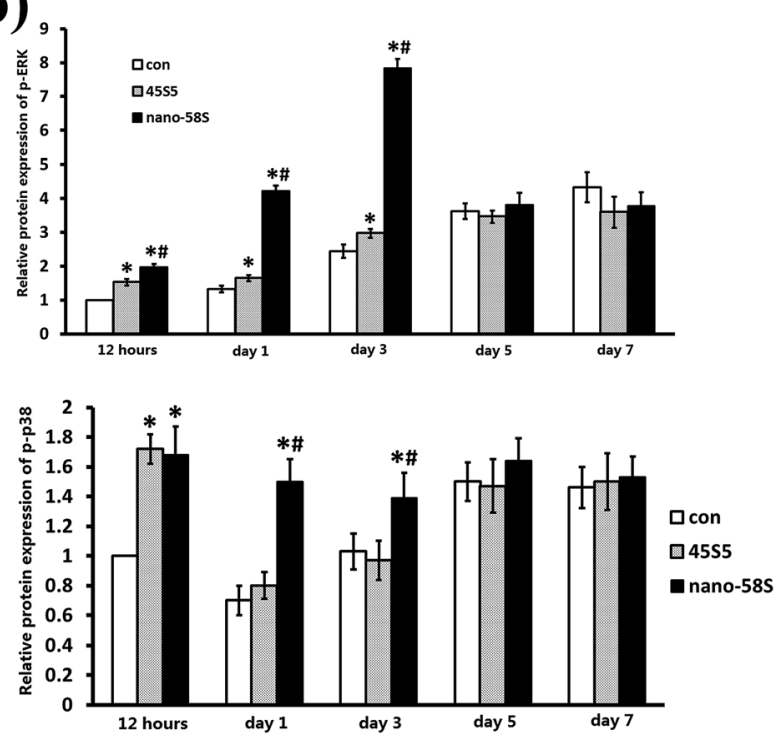

Fig. 4 (a) Western blot of ERK, p38 and JNK of MG- 63 cells cultured in, every three bands, left to right, the control medium, 45S5 BG extraction and nano-58S BG extraction, respectively, at 12 hours, and 1, 3, 5 and 7 days; and (b) quantification of the expression of phosphorylated ERK and phosphorylated p38 using ImageJ 1.38 software.

significant difference between the control, nano-58S and 45S5 groups with or without SB203580. Col I expression was similar in all three groups with SB203580 and was significantly lower than in the control group without SB203580. The gene expressions of Runx2 and OCN in the control, nano-58S and 45S5 groups with SB203580 were similar to the control group without SB203580, indicating no significant gene activation (Fig. 5(b)).

\section{Discussion}

The MG-63 cell line has been used in several studies due to its ideal osteoblastic characteristics, including the expression of BMP, ALP, Runx2 and OCN. In this study we focussed on ALP, Col I, Runx2 and OCN to observe the osteogenic gene activation effects of BG. ALP is the marker of the mature osteoblastic phenotype and extracellular matrix maturation. ${ }^{26} \mathrm{Col} \mathrm{I}$ is the 
(a)
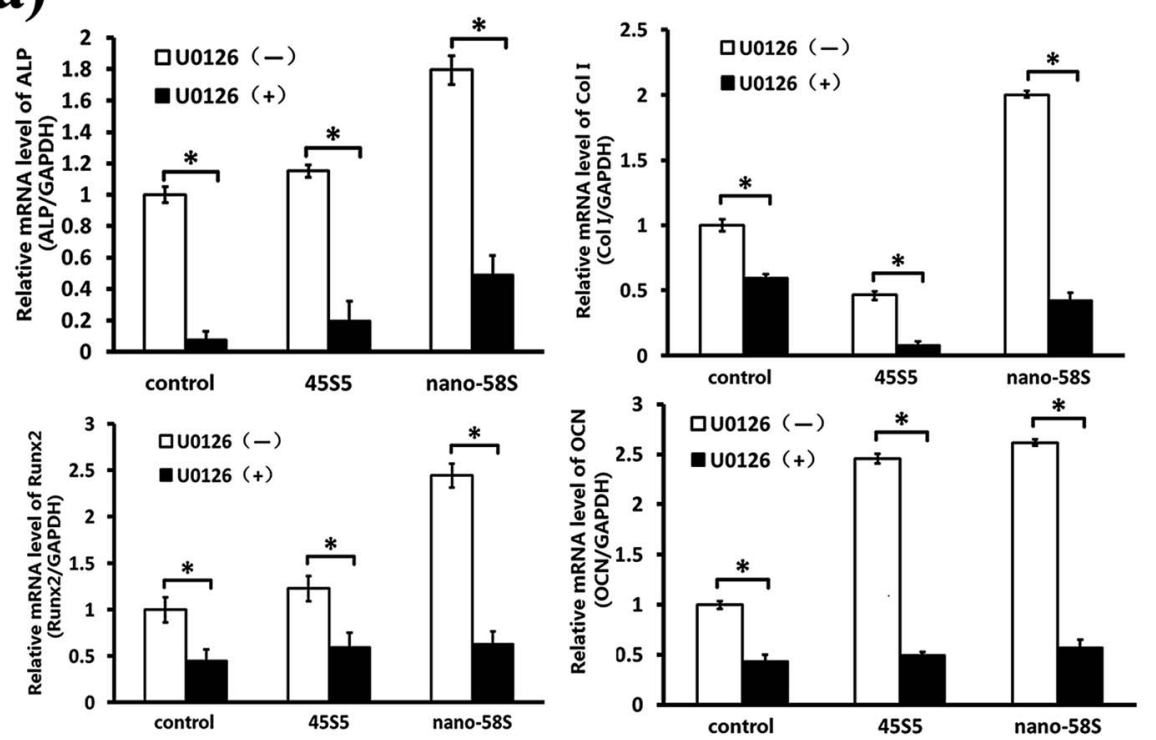

(b)
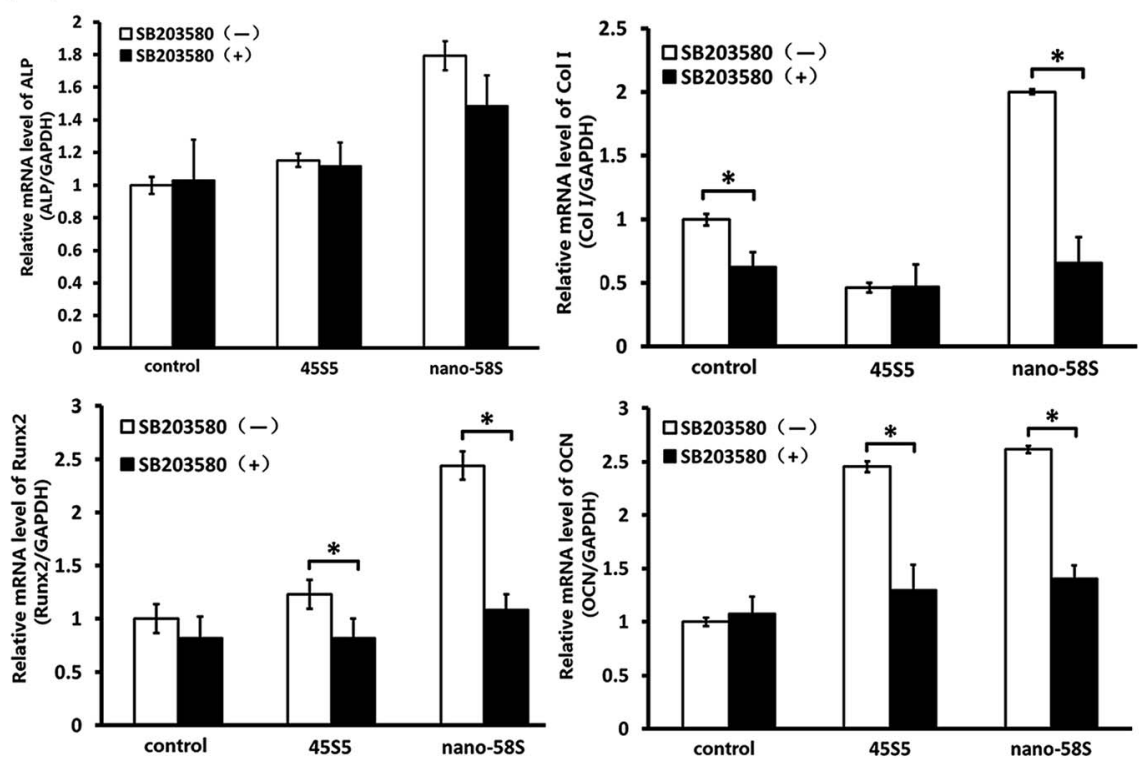

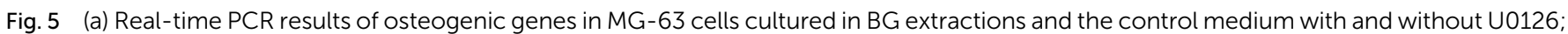

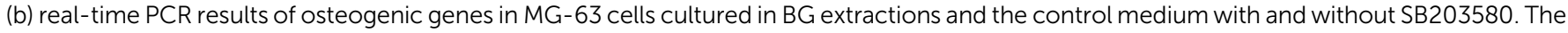

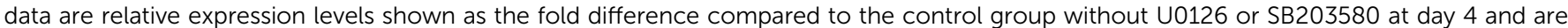

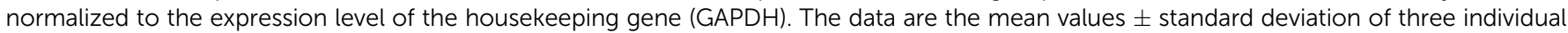

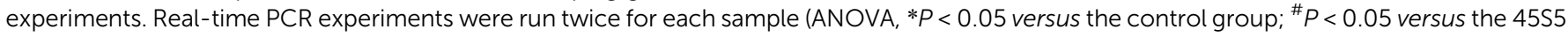
group).

predominant collagen in bone and the structural framework of matrices for inorganic deposition. ${ }^{27}$ Runx 2 is an essential transcript factor and master gene of osteoblast differentiation, and regulates the expression of downstream transcript factors and osteogenic markers. ${ }^{28}$ As a late osteoblast differentiation marker, OCN can bond $\mathrm{Ca}$ with bone matrix and induce mineralized nodule formation. ${ }^{29}$ The results of our study showed that nano-58S and 45S5 extractions enhanced these gene expressions. The positive Runx2 and OCN protein expressions, the increased protein secretion of Col I and OCN, as well as the matrix mineralization verified the genes' activation. According to the ELISA results, the increased Col I and OCN protein expression suggested that nano-58S BG and 45S5 BG promoted the early stages of bone matrix formation and mineralization in the MG-63 cells.

Previous studies have found that higher concentrations of $\mathrm{Si}$ and $\mathrm{Ca}$, a suitable $\mathrm{CaO} / \mathrm{SiO}_{2}$ ratio and low concentrations of $\mathrm{P}$ can enhance an osteoblasts' proliferation and 
differentiation..$^{\mathbf{3 0 , 3 1}}$ In agreement with the previous studies, and compared to 45S5 extractions, the possible reasons leading to the better gene activation of nano-58S BG may be the higher specific surface, better capacity for releasing ions of the particulates, and lower concentration of $\mathrm{P}$ in the extraction. However, besides the ion releasing capacity, there are several other features, such as composition, particle size, surface topography, degradation rate, and so on, which determine the bioactivity of bioactive glasses. Although we focused on the ionic production features of BG in this study, we speculated that both the nano-particle and the chemistry of the material enhanced the bioactivity of the nano-58S bioactive glass. To discern the effect of each feature on the BG's bioactivity, a series of studies are needed in the future.

We hypothesized that osteogenic gene activation by BG is transduced through the MAPK signalling cascades into the cell's nucleus and the response genes. The results of our study showed that the ERK and p38 pathways were activated, but no change in the JNK pathway was seen throughout the 7 days of observation. These results suggest that the ERK and p38 pathways may be involved in BG-induced osteogenic gene activation.

To find out whether ERK and p38 play a role in signaling transduction and gene expression regulation, we blocked the two pathways. MG-63 cells treated with U0126 showed inhibition of the normal expression of ALP, Col I, Runx2 and OCN. The gene activation of Col I, Runx 2 and OCN by BG was also blocked. Blocking the p38 pathway showed no effect on the normal expression of Runx2 and OCN but the genes' activation by BG was blocked. The up-regulation of ALP by BG showed the same trend with or without SB203580. Inhibition of Col I normal expression was found in all three groups with SB203580. These results indicated that the ERK and p38 pathways play a role in BG-induced gene activation. Based on the results that blocking the ERK pathway inhibited the osteogenic genes' normal expression and the BG's gene activation, while blocking the p38 pathway only blocked the BG's gene activation, it may be that the ERK pathway plays a more important role compared to the p38 pathway.

Several previous studies suggested participation of MAPKs in BG-induced gene activation based on the gene expression change of MAPK members. In agreement with previous studies, the current study focused on the involvement of MAPKs and their phosphorylation and verified their signaling transducing effect for the first time. Previous studies have found that the ERK pathway regulates the proliferation and differentiation of mesenchymal stem cells. ${ }^{32}$ Specifically, ERK pathways can activate the transcription of Runx2 during osteoblast differentiation. ${ }^{33,34}$ As the "master gene" for osteogenesis, Runx2 also regulates the expression of non-collagenous proteins such as OCN and BSP. According to the previous studies and the results of the current study, we propose that BG extraction activates MAPK pathways, specifically the ERK and p38 pathways, to transduce extracellular ionic stimulation into the cell nucleus, and activate the expression of Runx2. Then Runx2 regulates osteoblast differentiation, the expression of OCN and finally mineralization (Fig. 6).

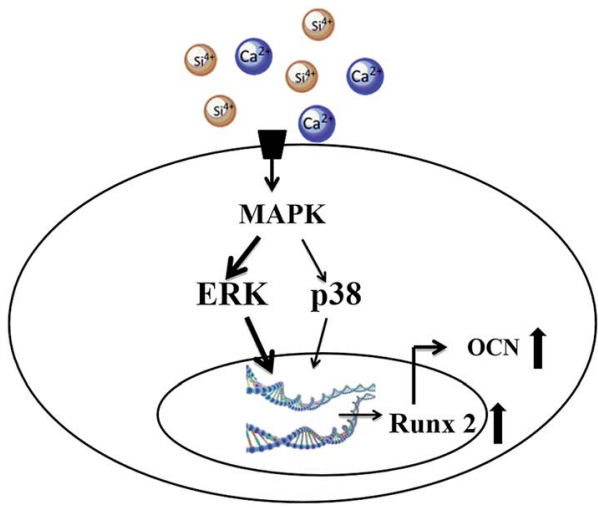

Fig. 6 Schematic of the cell signaling pathway for the activation of osteogenic gene expression by bioactive glasses.

\section{Conclusion}

The present study verified that the MAPK pathway is involved and plays an important part in BG's osteogenic gene activation. The effects of the novel nano-sized 58S BG on osteogenic genes up-regulation and MAPK phosphorylation are better than the traditional 45S5 BG.

\section{Acknowledgements}

This work was financially supported by the National Science Foundation of China (Project 51372005) and Peking University School of Stomatology (PKUSS20140101).

\section{References}

1 L. L. Hench, I. D. Xynos and J. M. Polak, Bioactive glasses for in situ tissue regeneration, J. Biomater. Sci., Polym. Ed., 2004, 15, 543-562.

2 G. C. Keles, B. O. Cetinkaya, D. Albayrak, H. Koprulu and G. Acikgoz, Comparison of platelet pellet and bioactive glass in periodontal regenerative therapy, Acta Odontol. Scand., 2006, 64, 327-333.

3 S. Mistry, D. Kundu, S. Datta and D. Basu, Comparison of bioactive glass coated and hydroxyapatite coated titanium dental implants in the human jaw bone, Aust. Dent. J., 2011, 56, 68-75.

4 F. Carinci, A. Palmieri, M. Martinelli, V. Perrotti, A. Piattelli, G. Brunelli, et al., Genetic portrait of osteoblast-like cells cultured on PerioGlas, J. Oral. Implantol., 2007, 33, 327-333.

5 J. Moura, L. N. Teixeira, C. Ravagnani, O. Peitl, E. D. Zanotto, M. M. Beloti, et al., In vitro osteogenesis on a highly bioactive glass-ceramic (Biosilicate), J. Biomed. Mater. Res., Part A, 2007, 82, 545-557.

6 J. Y. Sun, Y. S. Yang, J. Zhong and D. C. Greenspan, The effect of the ionic products of Bioglass dissolution on human osteoblasts growth cycle in vitro, J. Tissue Eng. Regener. Med., 2007, 1, 281-286.

7 T. J. Brunner, R. N. Grass and W. J. Stark, Glass and Bioglass nanopowders by flame synthesis, Chem. Commun., 2006, 13, 1384-1386. 
8 S. K. Misra, D. Mohn, T. J. Brunner, W. J. Stark, S. E. Philip, I. Roy, et al., Comparison of nanoscale and microscale bioactive glass on the properties of $\mathrm{P}(3 \mathrm{HB}) /$ Bioglass composites, Biomaterials, 2008, 29, 1750-1761.

9 J. R. Jones, New trends in bioactive scaffolds: the importance of nanostructure, J. Eur. Ceram. Soc., 2009, 29, 1275-1281.

10 I. D. Xynos, M. V. Hukkanen, J. J. Batten, L. D. Buttery, L. L. Hench and J. M. Polak, Bioglass 45S5 stimulates osteoblast turnover and enhances bone formation in vitro: implications and applications for bone tissue engineering, Calcif. Tissue Int., 2000, 67, 321-329.

11 I. D. Xynos, A. J. Edgar, L. D. Buttery, L. L. Hench and J. M. Polak, Gene-expression profiling of human osteoblasts following treatment with the ionic products of Bioglass 45S5 dissolution, J. Biomed. Mater. Res., 2001, 55, 151-157.

12 R. C. Bielby, I. S. Christodoulou, R. S. Pryce, W. J. P. Radford, L. L. Hench and J. M. Polak, Time- and concentrationdependent effects of dissolution products of $58 \mathrm{~S}$ sol-gel bioactive glass on proliferation and differentiation of murine and human osteoblasts, Tissue Eng., 2004, 10, 1018-1026.

13 Z. Yao and R. Seger, The ERK signaling cascade-views from different subcellular compartments, BioFactors, 2009, 35, 407-416.

14 M. A. Bogoyevitch, K. R. W. Ngoei, T. T. Zhao, Y. Y. C. Yeap and D. C. H. Ng, c-Jun N-terminal kinase (JNK) signaling: recent advances and challenges, Biochim. Biophys. Acta, Proteins Proteomics, 2010, 1804, 463-475.

15 Y. Obara and N. Nakahata, The signaling pathway leading to extracellular signal-regulated kinase 5 (ERK5) activation via G-proteins and ERK5-dependent neurotrophic effects, Mol. Pharmacol., 2010, 77, 10-16.

16 I. Christodoulou, L. D. Buttery, G. Tai, L. L. Hench and J. M. Polak, Characterization of human fetal osteoblasts by microarray analysis following stimulation with $58 \mathrm{~S}$ bioactive gel-glass ionic dissolution products, J. Biomed. Mater. Res., Part B, 2006, 77, 431-446.

17 A. Y. Au, R. Y. Au, J. L. Demko, R. M. McLaughlin, B. E. Eves and C. G. Frondoza, Consil bioactive glass particles enhance osteoblast proliferation and selectively modulate cell signaling pathways in vitro, J. Biomed. Mater. Res., Part A, 2010, 94, 380-388.

18 M. Ding, X. L. Shi, Z. G. Dong, F. Chen, Y. J. Lu, V. Castranova, et al., Freshly fractured crystalline silica induces activator protein-1 activation through ERKs and p38 MAPK, J. Biol. Chem., 1999, 274, 30611-30616.

19 M. L. Major, H. S. Cheung and R. P. Misra, Basic calcium phosphate crystals activate c-fos expression through a Ras/ ERK dependent signaling mechanism, Biochem. Biophys. Res. Commun., 2007, 355, 654-660.

20 S. Canadillas, R. Canalejo, M. E. Rodriguez-Ortiz, J. M. Martinez-Moreno, J. C. Estepa, R. Zafra, et al., Upregulation of parathyroid VDR expression by extracellular calcium is mediated by ERK1/2-MAPK signaling pathway, Am. J. Physiol. Ren. Physiol., 2010, 298, F1197-F1204.

21 X. H. Rausch-Fan, Z. Qu, M. Wieland, et al., Differentiation and cytokine synthesis of human alveolar osteoblasts compared to osteoblast-like cells (MG63) in response to titanium surfaces, Dent. Mater., 2008, 24, 102-110.

22 J. Clover and M. Gowen, Are MG-63 and HOS TE85 human osteosarcoma cell lines representative models of the osteoblastic phenotype?, Bone, 1994, 15, 585-591.

23 X. F. Chen, B. Lei, Y. J. Wang and N. Zhao, Morphological control and in vitro bioactivity of nanoscale bioactive glasses, J. Non-Cryst. Solids, 2009, 355, 791-796.

24 B. Lei, X. F. Chen, X. Han and J. A. Zhou, Versatile fabrication of nanoscale sol-gel bioactive glass particles for efficient bone tissue regeneration, J. Mater. Chem., 2012, 22, 1690616913.

25 X. F. Chen, Y. C. Meng, Y. L. Li and N. R. Zhao, Investigation on bio-mineralization of melt and sol-gel derived bioactive glasses, Appl. Surf. Sci., 2008, 255, 562-564.

26 M. D. Mckee, Extracellular Matrix Proteins, Alkaline Phosphatase and Pyrophosphate as Molecular Determinants of Bone, Tooth, Kidney and Vascular Calcification, AIP Conf. Proc., 2008, 1049, 25-32.

27 A. L. Boskey, T. M. Wright and R. D. Blank, Collagen and bone strength, J. Bone Miner. Res., 1999, 14, 330-335.

28 T. Komori, H. Yagi, S. Nomura, A. Yamaguchi, K. Sasaki, K. Deguchi, et al., Targeted disruption of Cbfa1 results in a complete lack of bone formation owing to maturational arrest of osteoblasts, Cell, 1997, 89, 755-764.

29 C. Chenu, S. Colucci, M. Grano, P. Zigrino, R. Barattolo, G. Zambonin, et al., Osteocalcin induces chemotaxis, secretion of matrix proteins, and calcium-mediated intracellular signaling in human osteoclast-like cells, $J$. Cell Biol., 1994, 127, 1149-1158.

30 S. Lossdorfer, Z. Schwartz, C. H. Lohmann, D. C. Greenspan, D. M. Ranly and B. D. Boyan, Osteoblast response to bioactive glasses in vitro correlates with inorganic phosphate content, Biomaterials, 2004, 25, 2547-2555.

31 M. Karpov, M. Laczka, P. S. Leboy and A. M. Osyczka, Sol-gel bioactive glasses support both osteoblast and osteoclast formation from human bone marrow cells, $J$. Biomed. Mater. Res., Part A, 2008, 84, 718-726.

$32 \mathrm{~W}$. Zhang and H. T. Liu, MAPK signal pathways in the regulation of cell proliferation in mammalian cells, Cell Res., 2002, 12, 9-18.

33 G. Z. Xiao, D. Jiang, P. Thomas, M. D. Benson, K. L. Guan, G. Karsenty, et al., MAPK pathways activate and phosphorylate the osteoblast-specific transcription factor, Cbfa1, J. Biol. Chem., 2000, 275, 4453-4459.

34 S. W. Kim, A. M. Muise, P. J. Lyons and H. S. Ro, Regulation of adipogenesis by a transcriptional repressor that modulates MAPK activation, J. Biol. Chem., 2001, 276, 10199-10206. 